\title{
Inequalities for second-order Riesz transforms associated with Bessel expansions \\ by
}

\section{Adam OSĘKOWSKI}

Presented by Rafat LATAEA

Summary. The paper contains the proofs of $L^{p}$, logarithmic and weak-type estimates for the second-order Riesz transforms arising in the context of multidimensional Bessel expansions. Using a novel probabilistic approach, which rests on martingale methods and the representation of Riesz transforms via associated Bessel-heat processes, we show that these estimates hold with constants independent of the dimension.

1. Introduction. One of the basic examples of Calderón-Zygmund singular integral operators in $\mathbb{R}^{d}$ are the so-called Riesz transforms, given by

$$
R_{j} f(x)=\frac{\Gamma\left(\frac{d+1}{2}\right)}{\pi^{\frac{d+1}{2}}} \text { p.v. } \int_{\mathbb{R}^{d}} \frac{x_{j}-y_{j}}{|x-y|^{d+1}} f(y) \mathrm{d} y, \quad j=1, \ldots, d .
$$

These operators and their second-order analogues (linear combinations of expressions of the form $\left.R_{j} R_{k}, j, k=1, \ldots, d\right)$ play an important role in harmonic analysis and the theory of PDEs. In particular, it is well-known that tight information about the norms of these objects, considered as operators on various function spaces, can be used in the study of regularity of certain elliptic partial differential equations. The literature on the subject is extremely vast and exploits a variety of different techniques coming from analysis and probability theory.

2020 Mathematics Subject Classification: Primary 42B15, 42B20; Secondary 42B10, $60 \mathrm{G} 44$.

Key words and phrases: Riesz transform, Hankel transform, Bessel expansion, martingale, differential subordination.

Received 2 March 2020; revised 5 October 2020.

Published online 15 October 2020. 
In the recent years, much effort has been made to extend the estimates for Riesz transforms to other contexts, in which $\mathbb{R}^{d}$, equipped with Lebesgue's measure, classical laplacian and classical Fourier transform, is replaced with some other homogeneous space possessing an appropriate differential operator. This problem has been studied in various setups, and the typical approach rests on careful examination of the pointwise behavior of certain associated kernels.

The purpose of this paper is to introduce a novel, probabilistic approach to the study of second-order Riesz transforms arising in the multidimensional setting of Bessel expansions [3, 4, 5]. To formulate our results, we need to introduce the basic setup which will be used throughout the paper. Let $d \geq 1$ be a fixed dimension and let $\lambda \in \mathbb{R}_{+}^{d}$ be a fixed multiindex. Consider the space $X=\mathbb{R}_{+}^{d}$ equipped with its Borel subsets and the product measure

$$
\mathrm{d} \mu_{\lambda}(x)=\prod_{j=1}^{d} x_{j}^{2 \lambda_{j}} \mathrm{~d} x_{j} .
$$

Then the Bessel differential operator, defined initially on $C_{c}^{\infty}\left(\mathbb{R}_{+}^{d}\right)$ by

$$
\Delta^{\lambda}=-\Delta-\sum_{j=1}^{d} \frac{2 \lambda_{j}}{x_{j}} \partial_{x_{j}},
$$

has a symmetric and nonnegative extension to $L^{2}\left(\mathbb{R}_{+}^{d}, \mathrm{~d} \mu_{\lambda}\right)$. It is easy to check that $\Delta^{\lambda}$ admits the decomposition $\Delta^{\lambda}=\sum_{j=1}^{d} \delta_{j}^{*} \delta_{j}$, where $\delta_{j}=\partial_{x_{j}}$ and $\delta_{j}^{*}=-\partial_{x_{j}}-2 \lambda_{j} / x_{j}$ is the formal adjoint of $\delta_{j}, j=1, \ldots, d$.

The following information on the spectral properties of $\Delta^{\lambda}$ will be needed later. For any $z \in \mathbb{R}_{+}^{d}$, consider the function

$$
\varphi_{z}^{\lambda}(x)=\prod_{j=1}^{d}\left(z_{j} x_{j}\right)^{-\lambda_{j}+1 / 2} J_{\lambda_{j}-1 / 2}\left(z_{j} x_{j}\right), \quad x \in \mathbb{R}_{+}^{d},
$$

where $J_{\nu}$ stands for the oscillating Bessel function of the first kind and order $\nu$ :

$$
J_{\nu}(z)=\sum_{k=0}^{\infty} \frac{(-1)^{k}}{k ! \Gamma(\nu+k+1)}(z / 2)^{2 k+\nu}
$$

(see 14] for more on Bessel functions). Then $\varphi_{z}^{\lambda}$ is an eigenfunction of the Bessel operator, corresponding to the eigenvalue $|z|^{2}: \Delta^{\lambda} \varphi_{z}^{\lambda}=|z|^{2} \varphi_{z}^{\lambda}$. Furthermore, the family $\left(\varphi_{z}^{\lambda}\right)_{z}$ can be used to introduce another important object, the (modified) Hankel transform $\mathcal{H}^{\lambda}$. This operator, defined initially on $C_{c}^{\infty}\left(\mathbb{R}_{+}^{d}\right)$, acts by the formula

$$
\mathcal{H}^{\lambda} f(x)=\int_{\mathbb{R}_{+}^{d}} \varphi_{x}^{\lambda}(y) f(y) \mu_{\lambda}(\mathrm{d} y),
$$


and plays the role of the Fourier transform from the Euclidean setting. It can be extracted from the reasoning of Betancor and Stempak [5] that $\mathcal{H}^{\lambda}$ extends to an isometry on $L^{2}\left(\mathrm{~d} \mu_{\lambda}\right)$ and satisfies $\mathcal{H}^{\lambda}=\left(\mathcal{H}^{\lambda}\right)^{-1}$. In addition, for any $f \in C_{c}^{\infty}\left(\mathbb{R}_{+}^{d}\right)$ and any $j=1, \ldots, d$, we have the identity

$$
\mathcal{H}^{\lambda}\left(\delta_{j}^{*} \delta_{j} f\right)(z)=\left|z_{j}\right|^{2} \mathcal{H}^{\lambda} f(z), \quad z \in \mathbb{R}_{+}^{d} .
$$

The Bessel heat semigroup $W_{t}^{\lambda}=\exp \left(-t \Delta^{\lambda}\right)$, corresponding to the generator $-\Delta^{\lambda}$, is given by

$$
W_{t}^{\lambda} f=\mathcal{H}^{\lambda}\left(e^{-t|\cdot|^{2}} \mathcal{H}^{\lambda} f\right)
$$

and admits the following representation. If $f \in L^{2}\left(\mathrm{~d} \mu_{\lambda}\right)$ and $x \in \mathbb{R}_{+}^{d}$, then

$$
W_{t}^{\lambda} f(x)=\int_{\mathbb{R}_{+}^{d}} W_{t}^{\lambda}(x, y) f(y) \mathrm{d} \mu_{\lambda}(y),
$$

where the kernel $W_{t}^{\lambda}(\cdot, \cdot)$ is given by the formula

$$
W_{t}^{\lambda}(x, y)=\frac{1}{(2 t)^{d}} \exp \left(-\frac{1}{4 t}\left(|x|^{2}+|y|^{2}\right)\right) \prod_{j=1}^{d}\left(x_{j} y_{j}\right)^{-\lambda_{j}+1 / 2} I_{\lambda_{j}-1 / 2}\left(\frac{x_{j} y_{j}}{2 t}\right) .
$$

Here $x, y \in \mathbb{R}_{+}^{d}, t>0$ and

$$
I_{\nu}(z)=\sum_{k=0}^{\infty} \frac{(z / 2)^{2 k+\nu}}{k ! \Gamma(\nu+k+1)}
$$

stands for the non-oscillating modified Bessel function of the first kind and order $\nu$ (see [14 for details).

We are ready to formulate the main results of this paper. In what follows, for any vector $a=\left(a_{1}, \ldots, a_{d}\right) \in \mathbb{C}^{d}$, the operator $T^{a, \lambda}$ is a Hankel multiplier with symbol $|\xi|^{-2} \sum_{j=1}^{d} a_{j}\left|\xi_{j}\right|^{2}$; that is, we have the identity

$$
\mathcal{H}^{\lambda}\left(T^{a, \lambda} f\right)(\xi)=|\xi|^{-2} \sum_{j=1}^{d} a_{j}\left|\xi_{j}\right|^{2} \mathcal{H}^{\lambda} f(\xi), \quad \xi \in \mathbb{R}_{+}^{d}
$$

It is easy to see (using 1.1), for example) that $T^{a, \lambda}$ can be expressed as a linear combination of second-order Bessel-Riesz transforms:

$$
T^{a, \lambda}=\sum_{j=1}^{d} a_{j}\left(R_{j}^{\lambda}\right)^{*} R_{j}^{\lambda}
$$

Here $R_{j}^{\lambda}$ stands for the first-order Riesz transform given by $R_{j}^{\lambda} f(x)=$ $\delta_{j} \mathcal{H}^{\lambda}\left(|\cdot|^{-1} \mathcal{H}^{\lambda} f\right)(x)$ and $\left(R_{j}^{\lambda}\right)^{*}$ is its formal adjoint, that is, $\left(R_{j}^{\lambda}\right)^{*} f(x)=$ $\mathcal{H}^{\lambda}\left(|\cdot|^{-1} \mathcal{H}^{\lambda}\left(\delta_{j}^{*} f\right)\right)(x)$. 
The primary goal of this paper is to show, using probabilistic methods, that the operators $T^{a, \lambda}$ are bounded on various classical spaces. Let us start with $L^{p}$-estimates.

TheOrem 1.1. Pick a sequence $a=\left(a_{1}, \ldots, a_{d}\right)$ such that $\left|a_{j}\right| \leq 1$, $j=1, \ldots, d$. Then for any $1<p<\infty$ we have

$$
\left\|T^{a, \lambda}\right\|_{L^{p}\left(\mathbb{R}_{+}^{d}, \mathrm{~d} \mu_{\lambda}\right) \rightarrow L^{p}\left(\mathbb{R}_{+}^{d}, \mathrm{~d} \mu_{\lambda}\right)} \leq p^{*}-1,
$$

where $p^{*}=\max \{p, p /(p-1)\}$.

In the boundary cases $p=1$ and $p=\infty$, we will establish the corresponding $L \log \mathrm{L}$ and exponential inequalities. It will be convenient to use the functions $\Phi(t)=e^{t}-1-t$ and $\Psi(t)=(t+1) \log (t+1)-t$, defined for $t \geq 0$.

TheOrem 1.2. Pick a sequence $a=\left(a_{1}, \ldots, a_{d}\right)$ such that $\left|a_{j}\right| \leq 1$, $j=1, \ldots, d$. Then for any $K>1$ and any Borel subset $A$ of $\mathbb{R}_{+}^{d}$ we have

$$
\int_{A}\left|T^{a, \lambda} f(x)\right| \mathrm{d} \mu_{\lambda}(x) \leq K \int_{\mathbb{R}_{+}^{d}} \Psi(|f(x)|) \mathrm{d} \mu_{\lambda}(x)+\frac{\mu_{\lambda}(A)}{2(K-1)} .
$$

Furthermore, if $\|f\|_{L^{\infty}\left(\mathbb{R}_{+}^{d}, \mathrm{~d} \mu_{\lambda}\right)} \leq 1$, then

$$
\int_{\mathbb{R}_{+}^{d}} \Phi\left(\left|T^{a, \lambda} f(x)\right|\right) \mathrm{d} \mu_{\lambda}(x) \leq \frac{1}{2 K(K-1)}\|f\|_{L^{1}\left(\mathbb{R}_{+}^{d}, \mathrm{~d} \mu_{\lambda}\right)} .
$$

We will also establish the corresponding weak-type bounds; for any $1<$ $p<\infty$, we will work with the norm

$$
\|f\|_{L^{p, \infty}\left(\mathbb{R}_{+}^{d}, \mathrm{~d} \mu_{\lambda}\right)}=\sup \left\{\frac{1}{\mu_{\lambda}(A)^{1-1 / p}} \int_{A}|f(x)| \mathrm{d} \mu_{\lambda}(x)\right\},
$$

where the supremum is taken over all Borel subsets $A$ of $\mathbb{R}_{+}^{d}$ satisfying $\mu_{\lambda}(A)>0$. We introduce the constants

$$
K_{p}= \begin{cases}{\left[\frac{1}{2} \Gamma\left(\frac{2 p-1}{p-1}\right)\right]^{(p-1) / p}} & \text { if } 1<p<2, \\ \left(p^{p-1} / 2\right)^{1 / p} & \text { if } p \geq 2 .\end{cases}
$$

Theorem 1.3. Assume that $1<p<\infty$ and let $a_{1}, \ldots, a_{d}$ be elements of the unit ball in $\mathbb{C}$. Then

$$
\left\|T^{a, \lambda}\right\|_{L^{p}\left(\mathbb{R}_{+}^{d}, \mathrm{~d} \mu_{\lambda}\right) \rightarrow L^{p, \infty}\left(\mathbb{R}_{+}^{d}, \mathrm{~d} \mu_{\lambda}\right)} \leq K_{p} .
$$

A few comments on the method of proof are in order. A classical argument used to establish results of the above type studies various delicate properties of the kernel $W^{\lambda}$. Our approach will be completely different and will exploit probabilistic methods: the above estimates will be deduced from some deep 
results from martingale theory. As a by-product, we obtain constants which do not depend on the dimension $d$ or the parameter $\lambda$.

The paper is organized as follows. In the next section we present the probabilistic facts which will be needed to establish the aforementioned inequalities. Section 3 links the probabilistic and analytic aspects of the paper, and is devoted to a martingale representation of the operators $T^{a, \lambda}$. In the final part we put all the facts together and establish Theorems 1.11 .3$.

2. Probabilistic background. As announced in the preceding section, our arguments depend heavily on probabilistic techniques. Let us introduce the necessary setup and notation.

Suppose that $(\Omega, \mathcal{F}, \mathbb{P})$ is a complete probability space, equipped with $\left(\mathcal{F}_{t}\right)_{t \geq 0}$, a nondecreasing family of sub- $\sigma$-fields of $\mathcal{F}$, such that $\mathcal{F}_{0}$ contains all the events of probability 0 .

Assume further that $X, Y$ are two adapted martingales taking values in a certain separable Hilbert space $(\mathcal{H},|\cdot|)$; with no loss of generality, we may put $\mathcal{H}=\ell^{2}$. As usual, we impose standard conditions on the trajectories of these processes: we assume that they are right-continuous and have limits from the left. Given $p \in[1, \infty]$, we will write $\|X\|_{p}$ for the $p$ th moment of $X$, given by $\|X\|_{p}=\sup _{t \geq 0}\left\|X_{t}\right\|_{p}$. The symbol $[X, Y]$ will stand for the quadratic covariance process of $X$ and $Y$. See e.g. Dellacherie and Meyer [8] for details in the case when the processes are real-valued, and extend the definition to the vector setting by $[X, Y]=\sum_{k=0}^{\infty}\left[X^{k}, Y^{k}\right]$, where $X^{k}, Y^{k}$ are the $k$ th coordinates of $X, Y$, respectively.

We will say that $Y$ is differentially subordinate to $X$ if the process $\left([X, X]_{t}-[Y, Y]_{t}\right)_{t \geq 0}$ is nonnegative and nondecreasing as a function of $t$. This notion appeared for the first time in the discrete-time setting in the works of Burkholder [6, 7]; the above continuous-time extension was introduced by Bañuelos and Wang [2] and Wang [13. The differential subordination implies many interesting inequalities between the martingales $X$ and $Y$; for an overview of the results, methods and much more, see the monograph by Osękowski [9].

The inequalities (1.3)- 1.6 will be deduced from their probabilistic counterparts. We start with the following $L^{p}$-estimate, proved by Burkholder [6] in the discrete-time setting and extended to the general context by Wang [13].

Theorem 2.1. Suppose that $Y$ is differentially subordinate to $X$. Then for any $1<p<\infty$ we have

$$
\|Y\|_{p} \leq\left(p^{*}-1\right)\|X\|_{p} .
$$

To show (1.5), we will need the following statement, established in [10]. Then (1.4) will be obtained with the use of a duality-type argument. 
TheOREM 2.2. Assume that $X, Y$ are $\mathcal{H}$-valued martingales such that $\|X\|_{\infty} \leq 1$ and $Y$ is differentially subordinate to $X$. Then for any $K>1$,

$$
\sup _{t \geq 0} \mathbb{E} \Phi\left(\left|Y_{t}\right| / K\right) \leq \frac{1}{2 K(K-1)}\|X\|_{1} .
$$

Finally, to prove (1.6), we will exploit the following fact from [11, which can be regarded as a dual to the weak-type inequalities between $X$ and $Y$.

THEOREM 2.3. Assume that $X, Y$ are $\mathcal{H}$-valued martingales such that $Y$ is differentially subordinate to $X$. Then for any $1<q<\infty$ and $p=q /(q-1)$,

$$
\|Y\|_{q}^{q} \leq K_{p}^{q}\|X\|_{1}\|X\|_{\infty}^{q-1}
$$

In the remainder of this section, we will provide some basic facts concerning Bessel processes. The interested reader is referred to [12, Chapter XI] for a systematic presentation of the subject.

Let $\beta=\left(\beta_{t}\right)_{t \geq 0}$ be a standard, one-dimensional Brownian motion. For every $\delta \geq 0$ and $x \geq 0$, the equation

$$
Z_{t}=x+2 \int_{0}^{t} \sqrt{Z_{s}} \mathrm{~d} \beta_{s}+\delta t
$$

has a unique strong solution, which is called the square of a $\delta$-dimensional Bessel process started at $x$ (and denoted by $\mathrm{BESQ}^{\delta}(x)$ ). For any $x \geq 0$, the square root of $\mathrm{BESQ}^{\delta}\left(x^{2}\right)$ is called a Bessel process of dimension $\delta$ started at $x$, and is denoted by $\operatorname{BES}^{\delta}(x)$. The family $\left(\operatorname{BES}^{\delta}(x)\right)_{x \geq 0}$ is a Markov family with density

$$
p_{t}^{\delta}(x, y)=t^{-1}(y / x)^{\delta / 2-1} y \exp \left(-\frac{x^{2}+y^{2}}{2 t}\right) I_{\delta / 2-1}\left(\frac{x y}{t}\right), \quad x, y>0 .
$$

Obviously, the function $W_{t}^{\lambda}(x, y)$ given by 1.2 is closely related to the product $\prod_{j=1}^{d} p_{2 t}^{2 \lambda_{j}+1}\left(x_{j}, y_{j}\right)$; both functions are essentially equal (the slight difference in the formulas comes from the fact that $W_{t}^{\lambda}$ is the density of the semigroup with respect to the measure $\mathrm{d} \mu_{\lambda}$, while 2.3 refers to Lebesgue measure).

3. Probabilistic representation of second-order Riesz transforms. Now we will explain how second-order Riesz transforms can be expressed in terms of stochastic integrals involving Bessel processes. Suppose that $f$ is a smooth, compactly supported, complex-valued function on $\mathbb{R}_{+}^{d}$ and let $U_{f}: \mathbb{R}_{+}^{d} \times[0, \infty) \rightarrow \mathbb{C}$ denote the Bessel-heat extension of $f: U_{f}(\cdot, 0)=f$ and, for any $x \in \mathbb{R}_{+}^{d}$ and $t>0$,

$$
U_{f}(x, t)=W_{t}^{\lambda} f(x)=\int_{\mathbb{R}_{+}^{d}} W_{t}^{\lambda}(x, y) f(y) \mathrm{d} \mu_{\lambda}(y),
$$


where the kernel $W_{t}^{\lambda}(\cdot, \cdot)$ is given by 1.2 . This extension is a $C^{\infty}$ function on $\mathbb{R}_{+}^{d} \times \mathbb{R}^{+}$and satisfies the PDE

$$
\Delta_{x}^{\lambda} U_{f}+\frac{\partial U_{f}}{\partial t}=0
$$

where $\Delta_{x}^{\lambda}$ is the Bessel differential operator applied to the $x$-coordinate. We will also need the following "square" regularity property of $U_{f}$. Note that $W_{t}^{\lambda}$ extends to a $C^{\infty}$ function on $\mathbb{R}^{d} \times \mathbb{R}^{d}$ with the use of the formula

$W_{t}^{\lambda}(x, y)$

$$
=\frac{1}{(2 t)^{d}} \exp \left(-\frac{1}{4 t}\left(|x|^{2}+|y|^{2}\right)\right) \prod_{j=1}^{d} \sum_{k=0}^{\infty} \frac{\left(x_{j} y_{j}\right)^{2 k}}{(4 t)^{2 k+\lambda_{j}-1 / 2} k ! \Gamma(k+\lambda+1 / 2)}
$$

and hence $W_{t}^{\lambda}(\cdot, y)$ is a smooth function of $x_{1}^{2}, \ldots, x_{d}^{2}$. Clearly, this property is inherited by the function $U_{f}(\cdot, t)$.

Now, for a fixed $x \in \mathbb{R}_{+}^{d}$, let $X^{x}=\left(X^{1, x_{1}}, X^{2, x_{2}}, \ldots, X^{d, x_{d}}\right)$ be the collection of independent processes such that for each $j=1, \ldots, d$, the coordinate $X^{j, x_{j}}$ is a Bessel process of dimension $2 \lambda_{j}+1$, started at $x_{j}$. For a given positive number $T$, we introduce the associated parabolic process $F=F(x ; T ; f)$ by

$$
F_{t}=U_{f}\left(X_{2 t}^{x}, T-t\right), \quad t \in[0, T] .
$$

The next step is to apply Itô's formula to $F$. We have the SPDEs $d\left(X^{j, x_{j}}\right)_{t}^{2}=$ $2 X_{t}^{j, x_{j}} \mathrm{~d} B_{t}^{j}+\left(2 \lambda_{j}+1\right) \mathrm{d} t$, where $B=\left(B^{1}, \ldots, B^{d}\right)$ is a Brownian motion in $\mathbb{R}^{d}$. Let us rewrite $F$ in the form $F_{t}=U_{f}\left(\sqrt{\left(X_{2 t}^{x}\right)^{2}}, T-t\right), t \in[0, T]$. Then Itô's formula, combined with 3.1 , yields

$$
F_{t}(x ; T ; f)=U_{f}(x, T)+\int_{0+}^{t} \nabla_{x} U_{f}\left(X_{2 s}^{x}, T-s\right) \mathrm{d} B_{2 s}, \quad t \in[0, T] .
$$

This in particular implies that the process $F$ is a continuous-path martingale. Note that $F$ is bounded, and hence square-integrable. It follows from classical facts from stochastic analysis that the quadratic variation of $F$ equals

$$
[F, F]_{t}=\left|U_{f}(x, T)\right|^{2}+2 \int_{0+}^{t}\left|\nabla_{x} U_{f}\left(X_{2 s}^{x}, T-s\right)\right|^{2} \mathrm{~d} s, \quad t \in[0, T] .
$$

The next step of the construction is to apply a certain transformation to $F$. Suppose that $\mathcal{A}$ is a diagonal $d \times d$ matrix with each entry in the unit ball of $\mathbb{C}$. Consider the associated transform of $F$, given by

$$
G_{t}=G_{t}(x ; T ; f ; \mathcal{A})=\int_{0+}^{t} \mathcal{A} \nabla_{x} U_{f}\left(X_{2 s}^{x}, T-s\right) \mathrm{d} B_{2 s}, \quad t \in[0, T] .
$$


Then $G$ is a square-integrable martingale, with quadratic covariance process equal to

$$
[G, G]_{t}=2 \int_{0+}^{t}\left|\mathcal{A} \nabla_{x} U_{f}\left(X_{2 s}^{x}, T-s\right)\right|^{2} \mathrm{~d} s, \quad t \in[0, T] .
$$

Since the operator norm of $\mathcal{A}$ does not exceed 1 , we see that $G$ is differentially subordinate to $F$ : the process

$$
\begin{aligned}
{[F, F]_{t} } & -[G, G]_{t} \\
& =\left|U_{f}(x, T)\right|^{2}+2 \int_{0+}^{t}\left(\left|\nabla_{x} U_{f}\left(X_{2 s}^{x}, T-s\right)\right|^{2}-\left|\mathcal{A} \nabla_{x} U_{f}\left(X_{2 s}^{x}, T-s\right)\right|^{2}\right) \mathrm{d} s
\end{aligned}
$$

is nonnegative and nondecreasing. We will show now that an appropriate projection of the process $G$ leads to a Hankel multiplier. To this end, observe that for any $h \in L^{2}\left(\mathbb{R}_{+}^{d}, \mathrm{~d} \mu_{\lambda}\right)$ we have, by the Schwarz inequality and Fubini's theorem (and the fact that $\mu_{\lambda}$ is the invariant measure for the Bessel process $X$ ),

$$
\begin{aligned}
\mathbb{E} & \int_{\mathbb{R}_{+}^{d}}\left|G_{T}(x ; T ; f ; \mathcal{A}) h\left(X_{2 T}^{x}\right)\right| \mathrm{d} \mu_{\lambda}(x) \\
\leq & \left(\int_{\mathbb{R}_{+}^{d}} \mathbb{E}\left|G_{T}(x ; T ; f ; \mathcal{A})\right|^{2} \mathrm{~d} \mu_{\lambda}(x)\right)^{1 / 2}\left(\int_{\mathbb{R}_{+}^{d}} \mathbb{E}\left|h\left(X_{2 T}^{x}\right)\right|^{2} \mathrm{~d} \mu_{\lambda}(x)\right)^{1 / 2} \\
= & \left(\int_{\mathbb{R}_{+}^{d}} \mathbb{E}[G(x ; T ; f ; \mathcal{A}), G(x ; T ; f ; \mathcal{A})]_{T} \mathrm{~d} \mu_{\lambda}(x)\right)^{1 / 2} \\
& \times\left(\mathbb{E} \int_{\mathbb{R}_{+}^{d}}\left|h\left(X_{2 T}^{x}\right)\right|^{2} \mathrm{~d} \mu_{\lambda}(x)\right)^{1 / 2} \\
\leq & \left(\int_{\mathbb{R}_{+}^{d}} \mathbb{E}[F(x ; T ; f), F(x ; T ; f)]_{T} \mathrm{~d} \mu_{\lambda}(x)\right)^{1 / 2}\|h\|_{L^{2}\left(\mathbb{R}_{+}^{d}, \mathrm{~d} \mu_{\lambda}\right)} \\
= & \left(\int_{\mathbb{R}_{+}^{d}} \mathbb{E}\left|F_{T}(x ; T ; f)\right|^{2} \mathrm{~d} \mu_{\lambda}(x)\right)^{1 / 2}\|h\|_{L^{2}\left(\mathbb{R}_{+}^{d}, \mathrm{~d} \mu_{\lambda}\right)} \\
= & \left(\int_{\mathbb{R}_{+}^{d}} \mathbb{E}\left|f\left(X_{2 T}^{x}\right)\right|^{2} \mathrm{~d} \mu_{\lambda}(x)\right)^{1 / 2}\|h\|_{L^{2}\left(\mathbb{R}_{+}^{d}, \mathrm{~d} \mu_{\lambda}\right)}=\|f\|_{L^{2}\left(\mathbb{R}_{+}^{d}, \mathrm{~d} \mu_{\lambda}\right)}\|h\|_{L^{2}\left(\mathbb{R}_{+}^{d}, \mathrm{~d} \mu_{\lambda}\right)} .
\end{aligned}
$$

Consequently, there is a unique function $g=S^{T, \mathcal{A}} f \in L^{2}\left(\mathbb{R}_{+}^{d}, \mathrm{~d} \mu_{\lambda}\right)$ defined through the bilinear form 


$$
\int_{\mathbb{R}_{+}^{d}} g(x) h(x) \mathrm{d} \mu_{\lambda}(x)=\mathbb{E} \int_{\mathbb{R}_{+}^{d}} G_{T}(x ; T ; f ; \mathcal{A}) h\left(X_{2 T}^{x}\right) \mathrm{d} \mu_{\lambda}(x)
$$

for $h \in L^{2}\left(\mathbb{R}_{+}^{d}, \mathrm{~d} \mu_{\lambda}\right)$. (Informally, we can treat $g(x)$ as the projection, or rather the "conditional expectation" $\mathbb{E}_{\mathcal{P}}\left[G_{T}(x ; T ; f ; \mathcal{A}) \mid X_{2 T}^{x}=x\right]$ with respect to the product, non-probability measure $\left.\mathcal{P}=\mathbb{P} \otimes \mu_{\lambda}\right)$.

We will prove now that $S^{T, \mathcal{A}}$ is a Hankel multiplier and identify the associated symbol. Using basic properties of stochastic integrals, we note that for any $x \in \mathbb{R}_{+}^{d}$,

$$
\begin{aligned}
\mathbb{E} G_{T}(x ; T ; f ; \mathcal{A}) h\left(X_{2 T}^{x}\right) \\
\quad=\mathbb{E} \int_{0+}^{T} \mathcal{A} \nabla_{x} U_{f}\left(X_{2 s}^{x}, T-s\right) \mathrm{d} B_{2 s} \int_{0+}^{T} \nabla_{x} U_{h}\left(X_{2 s}^{x}, T-s\right) \mathrm{d} B_{2 s} \\
\quad=2 \mathbb{E} \int_{0+}^{T}\left\langle\mathcal{A} \nabla_{x} U_{f}\left(X_{2 s}^{x}, T-s\right), \nabla_{x} U_{h}\left(X_{2 s}^{x}, T-s\right)\right\rangle \mathrm{d} s \\
\quad=2 \int_{0+\mathbb{R}_{+}^{d}}^{T}\left\langle\mathcal{A} \nabla_{x} U_{f}(y, T-s), \nabla_{x} U_{h}(y, T-s)\right\rangle p_{2 s}(x, y) \mathrm{d} y \mathrm{~d} s \\
=2 \int_{0+}^{T} \int_{\mathbb{R}_{+}^{d}}\left\langle\mathcal{A} \nabla_{x} U_{f}(y, T-s), \nabla_{x} U_{h}(y, T-s)\right\rangle W_{s}^{\lambda}(x, y) \mathrm{d} \mu_{\lambda}(y) \mathrm{d} s,
\end{aligned}
$$

where $p_{s}(x, y)=\prod_{j=1}^{d} p_{s}^{2 \lambda_{j}+1}\left(x_{j}, y_{j}\right)$ is the transition density of $X^{x}$. Therefore, by Fubini's theorem and the fact that $\int_{\mathbb{R}_{+}^{d}} W_{s}^{\lambda}(x, y) \mathrm{d} \mu_{\lambda}(x)=1$ for each $y$, we may write

$$
\begin{aligned}
& \int_{\mathbb{R}_{+}^{d}} S^{T, \mathcal{A}} f(x) h(x) \mathrm{d} \mu_{\lambda}(x) \\
& =2 \iint_{\mathbb{R}_{+}^{d}}^{T} \int_{0+\mathbb{R}_{+}^{d}}\left\langle\mathcal{A} \nabla_{x} U_{f}(y, T-s), \nabla_{x} U_{h}(y, T-s)\right\rangle W_{s}^{\lambda}(x, y) \mathrm{d} \mu_{\lambda}(x) \mathrm{d} s \mathrm{~d} \mu_{\lambda}(y) \\
& =2 \iint_{\mathbb{R}_{+}^{d}}^{T}\left\langle\mathcal{A} \nabla_{x} U_{f}(y, T-s), \nabla_{x} U_{h}(y, T-s)\right\rangle \mathrm{d} s \mathrm{~d} \mu_{\lambda}(y) \\
& =2 \int_{\mathbb{R}_{+}^{d}} \int_{0+}^{T} \sum_{j=1}^{d} a_{j j} U_{f}(y, T-s) \delta_{j}^{*} \delta_{j} U_{h}(y, T-s) \mathrm{d} s \mathrm{~d} \mu_{\lambda}(y) .
\end{aligned}
$$


In the last line we have used integration by parts. Now fix $\xi \in \mathbb{R}_{+}^{d}$ and put

$$
h(x)=\varphi_{\xi}^{\lambda}(x)=\prod_{j=1}^{d}\left(\xi_{j} x_{j}\right)^{-\lambda_{j}+1 / 2} J_{\lambda_{j}-1 / 2}\left(\xi_{j} x_{j}\right),
$$

the eigenfunction of $\Delta^{\lambda}$ corresponding to the eigenvalue $|\xi|^{2}$. Then

$$
U_{h}(x, t)=\varphi_{\xi}^{\lambda}(x) \exp \left(-t|\xi|^{2}\right)
$$

and hence $\delta_{j}^{*} \delta_{j} U_{h}(x, t)=\left|\xi_{j}\right|^{2} \varphi_{\xi}^{\lambda}(x) \exp \left(-t|\xi|^{2}\right)$. Plugging this in the formula above, we get

$$
\begin{aligned}
\mathcal{H}^{\lambda}\left(S^{T, \mathcal{A}} f\right)(\xi)=\int_{\mathbb{R}_{+}^{d}} S^{T, \mathcal{A}} f(x) h(x) \mathrm{d} x \\
=2 \sum_{j=1}^{d} a_{j j}\left|\xi_{j}\right|^{2} \int_{\mathbb{R}_{+}^{d}} \int_{0+}^{T} U_{f}(y, T-s) \varphi_{\xi}^{\lambda}(y) \exp \left(-(T-s)|\xi|^{2}\right) \mathrm{d} s \mathrm{~d} \mu_{\lambda}(y) \\
=2 \sum_{j=1}^{d} a_{j j}\left|\xi_{j}\right|^{2} \int_{0+}^{T} \exp \left(-(T-s)|\xi|^{2}\right) \mathcal{H}^{\lambda}\left(U_{f}(\cdot, T-s)\right) \mathrm{d} s .
\end{aligned}
$$

We have $\mathcal{H}^{\lambda}\left(U_{f}(\cdot, t)\right)(\xi)=e^{-t|\xi|^{2}} \mathcal{H}^{\lambda} f(\xi)$, which implies

$$
\begin{aligned}
\mathcal{H}^{\lambda}\left(S^{T, \mathcal{A}} f\right)(\xi) & =2 \sum_{j=1}^{d} a_{j j}\left|\xi_{j}\right|^{2} \mathcal{H}^{\lambda} f(\xi) \cdot \int_{0+}^{T} e^{-2(T-s)|\xi|^{2}} \mathrm{~d} s \\
& =\mathcal{H}^{\lambda}(f)(\xi) \frac{\langle\mathcal{A} \xi, \xi\rangle}{|\xi|^{2}}\left[1-e^{-2 T|\xi|^{2}}\right] .
\end{aligned}
$$

Thus, $S^{T, \mathcal{A}}$ is a Hankel multiplier with symbol $\langle\mathcal{A} \xi, \xi\rangle|\xi|^{-2}\left[1-e^{-T|\xi|^{2}}\right]$.

4. Proofs of Theorems 1.1 1.3. Equipped with the representation of the preceding section, we are ready for the proofs of the results announced in the introduction.

Proof of (1.3). By a straightforward approximation argument, it is enough to show that

$$
\int_{\mathbb{R}_{+}^{d}}\left|T^{a, \lambda} f\right|^{p} \mathrm{~d} \mu_{\lambda} \leq\left(p^{*}-1\right)^{p} \int_{\mathbb{R}_{+}^{d}}|f|^{p} \mathrm{~d} \mu_{\lambda}
$$

for any $f \in C_{c}^{\infty}\left(\mathbb{R}_{+}^{d}\right)$. Let $A$ be the diagonal matrix with $a_{1}, \ldots, a_{d}$ on the main diagonal, and let $F, G$ be the associated martingales introduced in the preceding section. We will combine the differential subordination of these processes with Theorem 2.1. To this end, recall that $q=p /(p-1)$ is the conjugate to $p$ and note that for any $h \in L^{2}\left(\mathbb{R}^{d}, \mathrm{~d} \mu_{\lambda}\right)$, 


$$
\begin{aligned}
\mathbb{E} \int_{\mathbb{R}_{+}^{d}}\left|G_{T}(x ; T ; f ; \mathcal{A}) h\left(X_{2 T}^{x}\right)\right| \mathrm{d} \mu_{\lambda}(x) \\
\quad \leq\left(\int_{\mathbb{R}_{+}^{d}} \mathbb{E}\left|G_{T}(x ; T ; f ; \mathcal{A})\right|^{p} \mathrm{~d} \mu_{\lambda}(x)\right)^{1 / p}\left(\int_{\mathbb{R}_{+}^{d}} \mathbb{E}\left|h\left(X_{2 T}^{x}\right)\right|^{q} \mathrm{~d} \mu_{\lambda}(x)\right)^{1 / q} \\
\leq\left(p^{*}-1\right)\left(\int_{\mathbb{R}_{+}^{d}} \mathbb{E}\left|F_{T}(x ; T ; f)\right|^{p} \mathrm{~d} \mu_{\lambda}(x)\right)^{1 / p}\left(\int_{\mathbb{R}_{+}^{d}} \mathbb{E}\left|h\left(X_{2 T}^{x}\right)\right|^{q} \mathrm{~d} \mu_{\lambda}(x)\right)^{1 / q} \\
\quad=\left(p^{*}-1\right)\|f\|_{L^{p}\left(\mathbb{R}_{+}^{d}, \mathrm{~d} \mu_{\lambda}\right)}\|h\|_{L^{q}\left(\mathbb{R}_{+}^{d}, \mathrm{~d} \mu_{\lambda}\right)},
\end{aligned}
$$

by Fubini's theorem. This, by the very definition of $S^{T, \mathcal{A}}$, implies

$$
\left\|S^{T, \mathcal{A}} f\right\|_{L^{p}\left(\mathbb{R}_{+}^{d}, \mathrm{~d} \mu_{\lambda}\right)} \leq\left(p^{*}-1\right)\|f\|_{L^{p}\left(\mathbb{R}_{+}^{d}, \mathrm{~d} \mu_{\lambda}\right)} .
$$

Now, let $T \rightarrow \infty$. Since $\mathcal{H}^{\lambda}$ is an isometry, we see that $S^{T, \mathcal{A}} f$ converges in $L^{2}\left(\mathbb{R}_{+}^{d}, \mathrm{~d} \mu_{\lambda}\right)$ to the function $S^{\mathcal{A}} f$, where $S^{\mathcal{A}}$ is the Hankel multiplier with symbol $\langle\mathcal{A} \xi, \xi\rangle /|\xi|^{2}$. Hence we can pick a sequence $T_{n}$ converging to infinity such that $S^{T_{n}, A} f$ converges to $S^{\mathcal{A}} f \mu_{\lambda}$-almost everywhere on $\mathbb{R}_{+}^{d}$. So, Fatou's lemma combined with 4.1 gives

$$
\left\|S^{A} f\right\|_{L^{p}\left(\mathbb{R}_{+}^{d}, \mathrm{~d} \mu_{\lambda}\right)} \leq\left(p^{*}-1\right)\|f\|_{L^{p}\left(\mathbb{R}_{+}^{d}, \mathrm{~d} \mu_{\lambda}\right)}
$$

and it remains to note that $S^{\mathcal{A}}$ coincides with $T^{a, \lambda}$.

Proof of 1.5). We may and do assume that $\|f\|_{L^{1}\left(\mathbb{R}^{d}, \mathrm{~d} \mu_{\lambda}\right)}<\infty$, since otherwise there is nothing to prove. We also know that $f$ is bounded by 1 , and hence, by the Hölder inequality, $f \in L^{2}\left(\mathbb{R}_{+}^{d}, \mathrm{~d} \mu_{\lambda}\right)$. Recall the functions $\Phi(t)=e^{t}-1-t$ and $\Psi(t)=(t+1) \log (t+1)-t$; one easily checks that $\Psi^{\prime}$ and $\Phi^{\prime}$ are inverse to each other, so

$$
a b \leq \Psi(a)+\Phi(b)
$$

for any nonnegative $a$ and $b$. Now, $f$ is bounded by 1 , so the martingale $F(x ; T ; f)$ also enjoys this property. Hence, by 4.2 ,

$$
\begin{array}{rl}
\mid \int_{\mathbb{R}_{+}^{d}} S^{T, \mathcal{A}} & f(x) h(x) \mathrm{d} \mu_{\lambda}(x)\left|\leq \mathbb{E} \int_{\mathbb{R}_{+}^{d}}\right| G_{T}(x ; T ; f ; \mathcal{A}) h\left(X_{2 T}^{x}\right) \mid \mathrm{d} \mu_{\lambda}(x) \\
& \leq K \int_{\mathbb{R}_{+}^{d}} \Psi\left(\left|h\left(X_{2 T}^{x}\right)\right|\right) \mathrm{d} \mu_{\lambda}(x)+K \int_{\mathbb{R}_{+}^{d}} \mathbb{E} \Phi\left(\left|G_{T}(x ; T ; f ; \mathcal{A})\right| / K\right) \mathrm{d} \mu_{\lambda}(x) \\
& \leq K \int_{\mathbb{R}_{+}^{d}} \Psi\left(\left|h\left(X_{2 T}^{x}\right)\right|\right) \mathrm{d} \mu_{\lambda}(x)+\frac{1}{2(K-1)} \int_{\mathbb{R}_{+}^{d}} \mathbb{E}\left|F_{T}(x ; T ; f)\right| \mathrm{d} \mu_{\lambda}(x) \\
& =K \int_{\mathbb{R}_{+}^{d}} \Psi\left(\left|h\left(X_{2 T}^{x}\right)\right|\right) \mathrm{d} \mu_{\lambda}(x)+\frac{1}{2(K-1)}\|f\|_{L^{1}\left(\mathbb{R}_{+}^{d}, \mathrm{~d} \mu_{\lambda}\right)}
\end{array}
$$


Here in the second step we have exploited the inequality (2.1), and the last identity follows from Fubini's theorem and the fact that $\mu_{\lambda}$ is the invariant measure for $X$. Now fix a positive number $M$ and consider the function

$$
h(x)=\frac{\overline{S^{T, \mathcal{A}} f(x)}}{\left|S^{T, \mathcal{A}} f(x)\right|}\left(\exp \left(\left|S^{T, \mathcal{A}} f(x)\right| / K\right)-1\right) \chi_{\left\{\left|S^{T, \mathcal{A}} f(x)\right| \leq M\right\}},
$$

with the convention $h(x)=0$ if $S^{T, \mathcal{A}} f(x)=0$. This function belongs to $L^{2}\left(\mathbb{R}_{+}^{d}, \mathrm{~d} \mu_{\lambda}\right)$, since $|h(x)| \leq L\left|S^{T, \mathcal{A}} f(x)\right|$ for some constant $L$ (here we use the presence of the characteristic function in the definition of $h$ ) and $S^{T, \mathcal{A}} f \in$ $L^{2}\left(\mathbb{R}_{+}^{d}, \mathrm{~d} \mu_{\lambda}\right)$ (which follows from (4.1) and $f \in L^{2}\left(\mathbb{R}_{+}^{d}, \mathrm{~d} \mu_{\lambda}\right)$ ). Plugging $h$ into the above chain of inequalities, we get

$$
\begin{aligned}
\int_{\mathbb{R}_{+}^{d}}\left|S^{T, \mathcal{A}} f(x)\right|\left(\exp \left(\frac{\left|S^{T, \mathcal{A}} f(x)\right|}{K}\right)-1\right) \chi_{\left\{\left|S^{T, \mathcal{A}} f(x)\right| \leq M\right\}} \mathrm{d} \mu_{\lambda}(x) \\
\leq K \int_{\mathbb{R}_{+}^{d}}\left[\exp \left(\frac{\left|S^{T, \mathcal{A}} f(x)\right|}{K}\right)\left(\frac{\left|S^{T, \mathcal{A}} f(x)\right|}{K}-1\right)+1\right] \chi_{\left\{\left|S^{T, \mathcal{A}} f(x)\right| \leq M\right\}} \mathrm{d} \mu_{\lambda}(x) \\
\quad+\frac{1}{2(K-1)}\|f\|_{L^{1}\left(\mathbb{R}_{+}^{d}, \mathrm{~d} \mu_{\lambda}\right)}
\end{aligned}
$$

This is equivalent to

$$
\int_{\mathbb{R}_{+}^{d}} \Phi\left(\frac{\left|S^{T, \mathcal{A}} f(x)\right|}{K}\right) \chi_{\left\{\left|S^{T, \mathcal{A}} f(x)\right| \leq M\right\}} \mathrm{d} \mu_{\lambda}(x) \leq \frac{1}{2 K(K-1)}\|f\|_{L^{1}\left(\mathbb{R}_{+}^{d}, \mathrm{~d} \mu_{\lambda}\right)},
$$

so letting $M \rightarrow \infty$ and using Fatou's lemma gives

$$
\int_{\mathbb{R}_{+}^{d}} \Phi\left(\frac{\left|S^{T, \mathcal{A}} f(x)\right|}{K}\right) \mathrm{d} \mu_{\lambda}(x) \leq \frac{1}{2 K(K-1)}\|f\|_{L^{1}\left(\mathbb{R}_{+}^{d}, \mathrm{~d} \mu_{\lambda}\right)} .
$$

It remains to let $T \rightarrow \infty$ and repeat the argument used above in the proof of the $L^{p}$ estimate.

Proof of 1.4. We will use duality. We have

$$
\int_{A}\left|T^{a, \lambda} f(x)\right| \mathrm{d} \mu_{\lambda}(x)=\int_{\mathbb{R}_{+}^{d}} T^{a, \lambda} f(x) \overline{\eta(x)} \mathrm{d} \mu_{\lambda}(x),
$$

where $\eta(x)=\chi_{A}(x) T^{a, \lambda} f(x) /\left|T^{a, \lambda} f(x)\right|$ (again with the convention $\eta(x)=0$ when $\left.T^{a, \lambda} f(x)=0\right)$. Applying the Hankel transform, we see that the last integral equals 


$$
\begin{aligned}
\int_{\mathbb{R}_{+}^{d}} \mathcal{H}^{\lambda}\left(T^{a, \lambda} f\right)(\xi) \overline{\mathcal{H}^{\lambda}(\eta)(\xi)} \mathrm{d} \mu_{\lambda}(\xi) & =\int_{\mathbb{R}_{+}^{d}} \frac{\langle\mathcal{A} \xi, \xi\rangle}{|\xi|^{2}} \mathcal{H}^{\lambda} f(\xi) \overline{\mathcal{H}^{\lambda}(\eta(\xi))} \mathrm{d} \mu_{\lambda}(\xi) \\
& =\int_{\mathbb{R}_{+}^{d}} \mathcal{H}^{\lambda} f(\xi) \overline{\mathcal{H}^{\lambda}\left(T^{\bar{a}, \lambda} \eta(\xi)\right)} \mathrm{d} \mu_{\lambda}(\xi) \\
& =\int_{\mathbb{R}_{+}^{d}} f(x) \overline{T^{\bar{a}, \lambda} \eta(x)} \mathrm{d} \mu_{\lambda}(x)
\end{aligned}
$$

where $A$ is the diagonal matrix with $a_{1}, \ldots, a_{n}$ on the main diagonal, and $\bar{a}$ stands for the conjugate sequence $\bar{a}_{1}, \ldots, \bar{a}_{n}$. Now we exploit the inequalities (1.5) and 4.2), together with the fact that $\eta$ is bounded by 1 . As a result, we get

$$
\begin{aligned}
\int_{A}\left|T^{a, \lambda} f(x)\right| \mathrm{d} \mu_{\lambda}(x) & \leq K \int_{\mathbb{R}_{+}^{d}} \Psi(|f(x)|) \mathrm{d} \mu_{\lambda}(x)+K \int_{\mathbb{R}_{+}^{d}} \Phi\left(\frac{\left|T^{\bar{a}, \lambda} \eta(x)\right|}{K}\right) \mathrm{d} \mu_{\lambda}(x) \\
& \leq K \int_{\mathbb{R}_{+}^{d}} \Psi(|f(x)|) \mathrm{d} \mu_{\lambda}(x)+\frac{1}{2(K-1)}\|\eta\|_{L^{1}\left(\mathbb{R}_{+}^{d}, \mathrm{~d} \mu_{\lambda}\right)} \\
& =K \int_{\mathbb{R}_{+}^{d}} \Psi(|f(x)|) \mathrm{d} \mu_{\lambda}(x)+\frac{\mu_{\lambda}(A)}{2(K-1)},
\end{aligned}
$$

which is the desired assertion.

Proof of 1.6. Arguing as in the proof of the $L^{p}$ estimate (1.3), we deduce the inequality

$$
\left\|T^{a, \lambda} f\right\|_{L^{q}\left(\mathbb{R}_{+}^{d}, \mathrm{~d} \mu_{\lambda}\right)}^{q} \leq K_{p}^{q}\|f\|_{L^{1}\left(\mathbb{R}_{+}^{d}, \mathrm{~d} \mu_{\lambda}\right)}\|f\|_{L^{\infty}\left(\mathbb{R}_{+}^{d}, \mathrm{~d} \mu_{\lambda}\right)}^{q-1}
$$

from 2.2. Now, fix an arbitrary $f \in L^{p}\left(\mathbb{R}_{+}^{d}, \mathrm{~d} \mu_{\lambda}\right)$ and a Borel set $A \subset \mathbb{R}_{+}^{d}$. As in the proof of (1.4), we write

$$
\int_{A}\left|T^{a, \lambda} f(x)\right| \mathrm{d} \mu_{\lambda}(x)=\int_{\mathbb{R}_{+}^{d}} T^{a, \lambda} f(x) \overline{\eta(x)} \mathrm{d} \mu_{\lambda}(x)=\int_{\mathbb{R}_{+}^{d}} f(x) \overline{T^{\bar{a}, \lambda} \eta(x)} \mathrm{d} \mu_{\lambda}(x),
$$

where $\eta(x)=\chi_{A}(x) T^{a, \lambda} f(x) /\left|T^{a, \lambda} f(x)\right|$. This implies, by Hölder's inequality and 4.3),

$$
\begin{aligned}
& \int_{A}\left|T^{a, \lambda} f(x)\right| \mathrm{d} \mu_{\lambda}(x) \leq\|f\|_{L^{p}\left(\mathbb{R}_{+}^{d}, \mathrm{~d} \mu_{\lambda}\right)}\left\|T^{\bar{a}, \lambda} \eta\right\|_{L^{q}\left(\mathbb{R}_{+}^{d}, \mathrm{~d} \mu_{\lambda}\right)} \\
& \leq K_{p}\|f\|_{L^{p}\left(\mathbb{R}_{+}^{d}, \mathrm{~d} \mu_{\lambda}\right)}\|\eta\|_{L^{1}\left(\mathbb{R}_{+}^{d}, \mathrm{~d} \mu_{\lambda}\right)}^{1 / q}\|\eta\|_{L^{\infty}\left(\mathbb{R}_{+}^{d}, \mathrm{~d} \mu_{\lambda}\right)}^{1-1 / q} \\
& \leq K_{p}\|f\|_{L^{p}\left(\mathbb{R}_{+}^{d}, \mathrm{~d} \mu_{\lambda}\right)} \mu_{\lambda}(A)^{1 / q} .
\end{aligned}
$$

This yields (1.6), since $A$ was arbitrary. 
Acknowledgements. The author would like to express his gratitude to the anonymous referee for the careful reading of the paper and several helpful suggestions. The research was supported by Narodowe Centrum Nauki (Poland), grant DEC-2014/14/E/ST1/00532.

\section{References}

[1] M. Abramowitz and I. A. Stegun (eds.), Handbook of Mathematical Functions with Formulas, Graphs and Mathematical Tables, reprint of the 1972 edition, Dover Publ., New York, 1992.

[2] R. Bañuelos and G. Wang, Sharp inequalities for martingales with applications to the Beurling-Ahlfors and Riesz transformations, Duke Math. J. 80 (1995), 575-600.

[3] J. J. Betancor, J. C. Fariña, D. Buraczewski, T. Martínez and J. L. Torrea, Riesz transforms related to Bessel operators, Proc. Roy. Soc. Edinburgh Sect. A 137 (2007), $701-725$.

[4] J. J. Betancor, J. C. Fariña, T. Martinez and L. Rodríguez-Mesa, Higher order Riesz transforms associated with Bessel operators, Ark. Mat. 46 (2008), 219-250.

[5] J. J. Betancor and K. Stempak, Relating multipliers and transplantation for FourierBessel expansions and Hankel transform, Tohoku Math. J. (2) 53 (2001), 109-129.

[6] D. L. Burkholder, Boundary value problems and sharp inequalities for martingale transforms, Ann. Probab. 12 (1984), 647-702.

[7] D. L. Burkholder, A sharp and strict $L^{p}$-inequality for stochastic integrals, Ann. Probab. 15 (1987), 268-273.

[8] C. Dellacherie and P.-A. Meyer, Probabilities and Potential B: Theory of Martingales, North-Holland, Amsterdam, 1982.

[9] A. Osękowski, Sharp Martingale and Semimartingale Inequalities, IMPAN Monografie Mat. 72, Birkhäuser, 2012.

[10] A. Osękowski, Logarithmic inequalities for Fourier multipliers, Math. Z. 274 (2013), 515-530.

[11] A. Osękowski, Weak type inequalities for Fourier multipliers with applications to Beurling-Ahlfors transform, J. Math. Soc. Japan 66 (2014), 745-764.

[12] D. Revuz and M. Yor, Continuous Martingales and Brownian Motion, 3rd ed., Springer, 1999.

[13] G. Wang, Differential subordination and strong differential subordination for continuous time martingales and related sharp inequalities, Ann. Probab. 23 (1995), 522-551.

[14] G. N. Watson, A Treatise on the Theory of Bessel Functions, Cambridge Univ. Press, Cambridge, 1966.

Adam Osękowski

Faculty of Mathematics, Informatics and Mechanics

University of Warsaw

Banacha 2

02-097 Warszawa, Poland

ORCID: 0000-0002-8905-2418

E-mail: ados@mimuw.edu.pl 